\title{
Tagungsbericht
}

\section{Jahrestagung der Gesellschaft für Technikgeschichte 2009}

\author{
"Geschichte(n) der Robotik" vom 22. bis 24. Mai 2009 in Offenbach \\ von Sonja Petersen und Melike Sahinol
}

Die Jahrestagung 2009 der Gesellschaft für Technikgeschichte (GTG) fand vom 22. bis 24. Mai 2009 in der Hochschule für Gestaltung in Offenbach zum Thema „Geschichte(n) der Robotik“ statt. Nicht nur Beiträge aus der Technikgeschichte, sondern auch aus anderen Fachgebieten, wie Soziologie, Informatik, Ingenieurwissenschaften, Medienwissenschaften und Japanologie waren vertreten. Nach der Begrüßung durch Bernd Kracke, Präsident der Hochschule für Gestaltung, und Martina Heßler, Vorsitzende der Gesellschaft für Technikgeschichte, umriss Catarina Caetano da Rosa für den Programmausschuss der GTG die Relevanz des Themas „Geschichte(n) der Robotik“ für die Technikgeschichte. Ziel der Tagung sei, das Verhältnis zwischen Mensch und Maschine darzustellen und zu analysieren. Die Geschichte ,selbsttätiger Maschinen“ kann bis in das antike Griechenland und das Wort „Robotik“ bis ins 14. Jahrhundert zurückgeführt werden. Jedoch wurde das Wort „Roboter“ erst in den 1920er Jahren durch Karel Capek populär, der den Begriff in seinem Drama „R.U.R.“ für selbstständige Maschinen (Roboter) „um Menschen die Arbeit zu erleichtern und abzunehmen“ einführte. So sind „Geschichte(n) der Robotik" für die Technikgeschichte von großer Bedeutung, wollen sie doch unter anderem „die Beziehung zwischen Mensch und Maschine verstehen“. Dabei gilt es zu untersuchen, in welchen Bereichen und für welche Aufgaben diese Maschinen eingesetzt wurden und welche Außenwirkung selbige auf den Menschen, die Gesellschaft und umgekehrt hatten.

Jan Müggenburg (Wien) sowie Eric Lettkemann und Martin Meister (beide Berlin) näherten sich „Wissensgeschichten der Robotik“. Ausgehend von Martin Caidins Roman Cyborg untersuchte Müggenburg in seinem Vortrag „Die Menschmaschine. Bionik und Kybernetik in Science and Fiction“ die Darstellung von Bionik in beiden Bereichen. Die Figur des Cyborg ging in den 1960er Jahren aus drei technologischen Wissensfiguren hervor: dem „prosthetic man“, dem Gliedmaßen durch Prothesen ersetzt wurden, dem „amplified man“, dessen technische Gliedmaßen die natürlichen in schweren Arbeiten unterstützten, und dem ,closed cycle man“, der Teil eines technischen Mechanismus war und sich nicht mehr anpassen musste. Gemeinsam war ihnen die Voraussetzung von Technologien, die kompatibel mit dem 
biologischen Organismus waren. Während der Cyborg als sich frei bewegendes, denkendes, fühlendes Wesen, welches die Technik nicht wahr nimmt, dargestellt wurde, bleibt beim Roboter die Technik immer präsent. Den Wandel der Roboter - ausgehend von der US-amerikanischen Militärforschung des Zweiten Weltkriegs bis heute - verfolgten Lettkemann und Meister in ihrem Beitrag zur Geschichte der Kybernetik ,Vom Flugabwehrgeschütz zum niedlichen Roboter. Zur kybernetischen Vorgeschichte der Robotik - und zur heutigen Bezugnahme auf diese Geschichte“. Im Zuge des sich vollziehenden Wandels der Roboter, veränderte sich auch die „Mensch-Maschine-Symbiose“ von Waffensystemen über Medizintechnik bis hin zu „niedlichen Robotern“, wie den künstlichen Schildkröten von William Grey Walters. Letztere sollten die Ergebnisse der Kybernetik spielerisch demonstrieren. Klare Traditionslinien in der Geschichte der Robotik wurden in der Diskussion als noch nicht besetzt angesehen. Es wurde deutlich, dass es noch keine Geschichte der Robotik gibt. Diese gelte es zu schreiben mit besonderer Berücksichtigung darauf, welche Geschichte(n) und Traditionslinien die Robotik sich selbst geben wird.

In der Sektion „Roboter im Einsatz“ wurden die Einsatzmöglichkeiten von Industrierobotern und deren Auswirkungen auf die Gesellschaft und Arbeit erörtert. Ralf Spicker (Ulm) rückte mit seinem Beitrag ,Zwischen Science Fiction-Vorstellungen und technisch-wissenschaftlichen Leitbildern. Ein Überblick über die Geschichte und (nahe?) Zukunft des Industrieroboters" das Verhältnis zwischen Industrieroboter und Mensch ins Zentrum seiner Ausführungen. Er zog die Entwicklungslinie von den Werkzeugmaschinen der Industriellen Revolution bis hin zum ersten Industrieroboter „Unimate" in der Gießerei von General Motors in den USA. Zunächst als Handhabungsgerät verwendet, entwickelte sich dieser zu einer Arbeitsmaschine, die sich durch eine hohe Wiederholungsgenauigkeit auszeichnete. Spicker stellte heraus, dass die Erwartung, Industrieroboter seien Jobkiller, so nicht eintraf. Auch heute würden nicht alle Aufgaben von Maschinen übernommen; menschliche Fähigkeiten und Wissen seien weiterhin von großer Bedeutung. Die gesellschaftlichen Auswirkungen des Einsatzes von Robotern erörterten auch Judith Igelsböck und Astrid Weiss (beide Salzburg) in ihrem Beitrag „Wenn Roboter in unseren Alltag einziehen - Akzeptanzfaktoren für soziale Interaktion mit Robotern", der erste Ergebnisse des EU-Projekts Robot@cwe präsentierte. Wie Spicker konstatierten sie die Angst der Menschen, durch Roboter ersetzt zu werden. Roboter können gleichwohl nicht überall eingesetzt werden, bräuchten doch die meisten Arbeiten mehr als einfache Handgriffe. Vielmehr sei davon auszugehen, dass Roboter vor allem für schmutzige, gefährliche und repetitive Arbeiten eingesetzt werden. Dass die prinzipielle Akzeptanz von Robotern nicht unbedingt zu ihrem erfolgreichen Einsatz führen muss, illustrierte Michael Schöneich (Königstein) in seinem Beitrag „Robotertechnik in der DDR von 1980 bis 1990“ zur Geschichte prozessflexibler und prozessspezifischer Industrieroboter in der 
DDR. Im Sachsenring in Zwickau wurde 1978 der erste Industrieroboter für das Decklackspritzen von Trabantkarosserien aus Norwegen eingesetzt. Sowohl nichtbetroffene Mitarbeiter, Führungskräfte, Leitungskräfte als auch Arbeiter wurden intensiv geschult, um mögliche Ängste vor den Robotern abzubauen. Trotz dieser Schulungen und guter technischer Innovationsleistungen blieb der betriebswirtschaftliche Erfolg aus und man konnte die technische Lücke zum Westen auf Dauer nicht schließen.

Roboter kamen jedoch nicht nur in der Industrie zum Einsatz, sondern auch im Alltag. In der entsprechenden Sektion „Serviceroboter. Herausforderungen im Alltag“" stellte Frank Dittmann (München) die Geschichte der Servicerobotik seit den 1960er Jahren in seinem Beitrag „Serviceroboter - Die Heinzelmännchen des 21. Jahrhunderts?“ dar. Zielten die frühen Serviceroboter, wie „Shakey“, auf eine Synergie verschiedener Bereiche künstlicher Intelligenz ab, konzentrieren sich aktuelle Forschungsprojekte auf die Bereiche Überwachung, Schutz und Inspektion sowie auf die Verbesserung von Konsumgütern wie Staubsauger, Rasenmäher oder Spielzeug, die als Produkte des Massenkonsums lukrativ sind. Dittmann streifte auch die Wechselwirkungen zwischen der Entwicklung der klassischen Industrierobotertechnik und ihren Modifikationen für spezifische Anwendungskontexte etwa im Bereich der Medizin als Operationsroboter. Abschließend thematisierte er Einsatzgebiete humanoider Roboter, z.B. Pflegeroboter, und wies darauf hin, dass gegen sie insbesondere die mangelnden sozialen Kontakte sprechen würden. In der Diskussion wurde darauf abgehoben, dass entscheidend für den Bau von Servicerobotern ihre Sinnhaftigkeit sei. So seien beispielsweise solche zu präferieren, die für die Räumung von Minenfeldern eingesetzt werden, obwohl sich hier angesichts begrenzter Stückzahlen die Frage nach dem ökonomischen Mehrwert stelle. Zudem wurde darauf hingewiesen, dass mit steigender „Autonomie des Roboters“ die Intervention durch den Menschen immer mehr gefragt wäre. Der Frage nach der Einsetzbarkeit humanoider Serviceroboter im Alltag ging auch Christian Schlette (Aachen) in seinem Vortrag „Humanoide Serviceroboter und die Komplexität des Alltags" nach. Er konstatierte, dass unser komplexer Alltag die Entwicklung von Robotern erschwere, da dessen Herausforderungen unter Laborbedingungen nur schwer abbildbar seien. Die Komplexität erfordere, Roboter mit „,intelligenten Algorithmen“, Sensoren und Aktoren zur Aufgabenplanung, Bewegungsplanung und Kollisionsvermeidung auszustatten. Schlette stellte verschiedene Probleme bei der Entwicklung solcher Algorithmen vor. In der anschließenden Diskussion wurde vor allem nach den Anforderungen an den Menschen, mit dem Roboter umzugehen, gefragt und angedeutet, dass Ingenieure zum Teil menschliche Handlungsabläufe mit der Entwicklung von Robotern „mitkonstruieren“.

In der Sektion „Roboter in den Medien“ beschäftigten sich drei Beiträge mit auf den ersten Blick ungewöhnlichen Quellen der Technikgeschichte. 
Stefan Stein (Paderborn) führte in seinem Vortrag „Robotik im Spiegel der Comics. Darstellung von Robotik und Künstlicher Intelligenz in Comics“ durch die bunte Welt des Genres, von Daniel Düsentrieb bis Knick Knatterton. Er analysierte die Darstellungsweise von Robotern, ihre Charakterisierung sowie ihr Verhältnis zu Menschen und entwickelte unterschiedliche Kategorien wie den guten Kumpel, den Mad Scientist, den Roboterdoppelgänger oder den Kampfroboter. Nach gezeichneten Bildern nahm Karsten Weber (Opole) die Teilnehmer mit auf eine Reise in die Welt der bewegten Bilder in seinem Beitrag „Von Gort zu Sonny. Die Darstellung von Robotern in ScienceFiction-Filmen als Geschichte der Emanzipation“. Er arbeitete heraus, wie sich die Figur des Roboters seit 1950 vom Werkzeug zum Akteur, über den gehorsamen Diener mit eingebauter Wertehierarchie, bis hin zu einem gegen die Menschen rebellierenden Bordcomputer wandelte. Den vorläufigen Endpunkt des Prozesses bildet der Film I, Robot aus dem Jahr 2004, der Roboter als vollständig selbstständige Akteure präsentierte. Beide Beiträge demonstrierten das Erkenntnispotenzial „unorthodoxer" Quellen für „Geschichte(n) der Robotik“. Mit gleicher Stoßrichtung plädierte auch Lisa Nocks (Newark, NJ) in ihrem Beitrag „Practical Matters in Robotics and Science Fiction“ für die Verwendung von Science-Fiction-Literatur als Quelle der Technikgeschichte. Solche Geschichten seien ein Raum, in dem große Probleme der Gesellschaft aufgewiesen und bearbeitet werden könnten, wenn dieses in der wirklichen Welt nicht möglich sei. Science Fiction sei ein Genre, das den Einfluss der Gesellschaft auf die Technik verdeutliche. Der Wert narrativer Erzählungen - seien es Comics, Filme oder Romane - für die Geschichtsschreibung wurde in der Diskussion bestätigt. Für „Geschichte(n) der Robotik" müssten in ihnen insbesondere Erkenntnisse zum Umgang der Gesellschaft mit sowie der Einfluss von Technik auf die Gesellschaft untersucht werden.

Die große Bedeutung von narrativen Figuren spiegelt sich vor allem in der Gesellschaft Japans wider, der die Sektion „Japan, Land der Roboter“ gewidmet war. Erich Pauer (Marburg) ging in seinem Vortrag „Japanische Automaten (karakuri ningyô). Vorläufer der modernen Roboter?“ der Frage nach, ob es eine direkte Verbindung zwischen den früheren Automaten, den figürlichen karakuri ningyô, aus der Edo-Zeit (1600-1867) und den heutigen humanoiden Robotern in Japan gibt. In der technikhistorischen Literatur Japans werde der „Karkuri-Meister“ Hisashige (1799-1881) als Bindeglied zwischen Tradition und Moderne angeführt, jedoch stellte dieser unterschiedlichste mechanische Apparate her. Insbesondere waren seine Kenntnisse der Mechanik und Verarbeitung verschiedener Werkstoffe von großer Bedeutung. Hervorzuheben sei nicht etwa die spezielle Bauweise der Automaten, sondern vor allem die Substitution von Metall durch Holz, die die „Weiterentwicklung" der Karakuri beeinflusste und als technischer Wissensbestand in die Moderne einfloss. Die edo-zeitlichen Automaten hätten nur bedingt 
Einfluss auf die Industrialisierung Japans gehabt, auch würden sie als Vorläufer moderner Roboter überschätzt. Nach der Suche der Ursprünge humanoider Roboter bot Kenji Ito (Hayama/Kanagawa) in seinem Beitrag „Before Astroboy: Robot discourse, nuke talks, and cultural meanings of science and technology in mid-20th century Japan“ einen Überblick über die kulturellen und politischen Konnotationen dieser maßgebenden Manga-Figur und ihren Einfluss auf die Akzeptanz von Robotern in der japanischen Gesellschaft. Anhand der Figur des Astro Boys und anderer Roboter-Images illustrierte Ito die soziokulturellen Bedeutungen von Wissenschaft und Technologie in Japan seit der Vorkriegszeit. In der ersten Episode von Astro Boy reflektierte sein Schöpfer TEZUKA Osamu die Kriegserfahrungen Japans. Allmählich entwickelte sich die Figur zum Sinnbild eines Supermenschen, mit sympathischen Zügen und einem übersteigerten Optimismus für die Wissenschaft und Technologie des Nachkriegsjapans. Sie steht für die Vision des zukünftigen Japans als Land technischer Utopien. Astro Boy begegnete den Teilnehmern auch in Cosima Wagners (Frankfurt a.M.) Beitrag „Von Astro Boy zu ASIMO? Einblicke in die japanische Wissenschaft vom Roboter", in dem sie sich ausgehend von dieser Figur mit den Charakteristika der japanischen Roboter beschäftigte. Sie betonte die ,traumorientierte Robotik“ Japans, die sich beispielsweise in ASIMO widerspiegele, der keinen Eigennutzen aufweise und für seine Entwickler bei Honda als „Image“-Produkt und PR-Figur fungiere. Durch die Präsentation seiner Roboter auf der EXPO 1970, die als Koexistenz der japanischen Gesellschaft aufgefasst werden können, versuchte Japan sein nationales Ansehen zu stärken. Wagner unterstrich, dass Roboter als „kulturelle Produktionen und Inszenierungen“ betrachtet werden müssten. Denn eine kulturwissenschaftliche Perspektive auf technische Artefakte wäre unabdingbar, um die soziokulturelle Einbettung von Technik sowie den prinzipiellen Verhandlungscharakter in deren Herstellungs- und Verwendungskontexten zu lokalisieren. Auch Hironori Matsuzaki (Oldenburg) nahm in seinem Beitrag zur „Entwicklung von humanoiden Robotern im Kulturvergleich - Europa und Japan. Eine wissenssoziologische Analyse der Grenzfragen der Mensch-Maschine-Beziehung“ die kulturelle Dimension von Technik auf. In Europa hätten Menschen eine normative und kognitive Sonderstellung (Menschenrechte, etc.), die als Fundament der Unterscheidung zwischen Mensch und Maschine anzusehen sei. Der zunehmende lebensweltliche Einsatz autonomer Roboter jedoch mache den Kontakt mit ihnen unvermeidlich und werfe die Frage auf, wie man mit Robotern umzugehen habe und ob gegebenenfalls humanoiden Robotern der Status einer sozialen Person zugeschrieben werden könne oder solle. In anthropozentrischen Gesellschaftsformen bilde eine solche Konzeptualisierung von Robotern, die die zugrundeliegende Mensch-Maschine-Differenz hinterfrage, ein grundlegendes Problem. In Japan dagegen würde dieses ethische Konfliktpotenzial kaum wahrgenommen. Man ginge von einer Koexistenz humanoider Robo- 
ter aus, die gestützt durch die buddhistisch-shintoistische egalitäre Denktradition eine Integration von Robotern in vielen Lebensbereichen akzeptiere und eine Bedeutungsverschiebung des sozialen Lebens zuließe. Alle drei Beiträge betonten die Bedeutung kultureller Faktoren in der Erforschung von Technik am Beispiel des Roboters und diskutierten Fragen der kulturwissenschaftlich orientierten Technikgeschichte.

Die GTG-Jahrestagung 2009 verstand es, die Brücke zur gegenwärtigen Robotikforschung mit einem Vortrag des Informatikers Sven Behnke (Bonn) zum Thema „Vorbild Mensch. Humanoide Roboter“ zu schlagen. In einer Abendveranstaltung im Offenbacher Klingspor Museum, gefördert durch das Amt für Wirtschaftsförderung Offenbach, stellte er die aktuelle Entwicklungsarbeit an humanoiden Robotern vor, die sich durch eine menschenähnliche Körperform, Verhalten und Sensoren auszeichneten. Bekannt sind vor allem die seit 1997 entwickelten Fußballroboter, die in internationalen Turnieren der RoboCup-Ligen gegeneinander antreten. Hieran anknüpfend, wenn auch in einer anderen Sektion, reflektierte Hans-Joachim Braun (Hamburg) in seinem Beitrag „Roboterfußball. Spielwiese wofür?“ historisch die Frage, welche Bedeutung Roboterwettkämpfe für die Entwicklungsarbeit und die Gesellschaft haben. Bereits in den 1920er Jahren beeinflusste der Taylorismus u.a. durch die Automatisierung von Laufwegen den Fußball. Durch die Optimierung von Laufwegen und Spieltaktiken, sowohl in Computerspielen als auch beim RoboCup, ergäben sich neue Möglichkeiten für die Entwicklung des „realen“ Fußballs. Das Automatisierungsparadigma sei bereits durch verschiedene Akteure (Trainer, Spieler etc.) im Bereich des Fußballs verinnerlicht worden. Doch müsse das Mensch-Maschine-Verhältnis neu gedacht werden. Lägen die Stärken der Roboter darin, keine Ermüdungserscheinungen zu zeigen und keine unnötigen Fouls zu begehen, müsse der Mensch sich auf die eigenen Stärken besinnen. So könne der Roboterfußball ein Vehikel sein, um über die Rolle des Schiedsrichters und einen humaneren Fußball nachzudenken.

Wettkämpfe zwischen Robotern gab es bereits in der Antike, wie Porfirio Silva (Lissabon) in seinem Vortrag „Fabulous races of human monsters and robots“ in der Sektion „Roboter als Alter Ego?“ darstellte. Er illustrierte, wie in der Antike Roboter zunächst als Devariationen des „Normalen“ als „fabulous races“ (märchenhafte Rassen) abgebildet und als „humanoide Monster“ thematisiert wurden. Silva sieht die Frage, was „humanoid“ ist, als zentral an, um zu klären, was wir unter humanoiden Robotern verstünden. Auf der einen Seite sei reale Menschenähnlichkeit wichtig, um Menschen zu verstehen. Auf der anderen Seite reagierten wir jedoch mit Abneigung auf Roboter mit allzu menschenähnlichen Zügen, und betrachteten sie als Monster. Die Frage nach der Identität von Robotern griff ebenfalls Jessica Heesen (Freiburg) in ihrem Beitrag „Der dezentrale Roboter und das postmoderne Subjekt" auf. Das Selbst würde durch soziale Interaktionen, durch Wechselverhältnisse zu Organisationsformen, sozialen Ordnungen und materialen Kon- 
texten entstehen. Der Aufbau von Identitäten würde in einer von Kommunikationstechniken dominierten Welt über elektronische Interaktionen mitbestimmt und sei von materialen Parametern abhängig. Mit der Ausweitung der intersubjektiven, elektronischen Kommunikation in der physischen Umgebung würde ein Alter-Ego im Kontext geschaffen und das Konzept einer reflexiven Identitätsausbildung normativ aufgeladen. Die ironische Wendung liege darin, dass der Kontext selbst zum expliziten Kommunikationspartner würde. Die Idee einer reflexiven Identitätsausbildung würde pragmatisch ersetzt durch adaptive Bildung von Nutzerstereotypen.

„Geschichte(n) der Robotik“ diskutierte die Entwicklung von Robotern auf unterschiedlichste Weise und schaffte so eine Verbindung zwischen technischen, gesellschaftlichen und kulturellen Aspekten des Einsatzes und Umgangs mit Robotern.

Traditionell wurden auch dieses Jahr zwei Exkursionen angeboten. Eine Gruppe konnte sich der Industriegeschichte der Stadt Rüsselsheim bei einem Stadtrundgang mit Besuch des Stadtmuseums nähern. Eine zweite Gruppe wurde durch das historische Opel-Altwerk in Rüsselsheim und seine leerstehenden Hallen geführt. Peter Schirmbeck erläuterte mit Hilfe historischer Fotografien die (Industrie-)Architektur und Anlage des Werkes sowie die technische Ausstattung der Hallen. Die Führung wurde abgerundet durch einige Ausführungen zu den modernen Industrierobotern, die in der heutigen Fertigung von Opel zum Einsatz kommen. Somit verband die GTGJahrestagung 2009 die wissenschaftliche Bearbeitung des Themenkomplexes "Geschichte(n) der Robotik“ und das physische Erleben bedeutender Industriestätten, in denen Menschen an und mit Maschinen arbeiteten.

Der Bericht wurde in gekürzter Form auch bei H-Soz-u-Kult und im ICOHTEC Newsletter veröffentlicht:

Tagungsbericht „Geschichte(n) der Robotik“, Jahrestagung der Gesellschaft für Technikgeschichte 2009, in: H-Soz-u-Kult, 5.8.2009 (http://hsozkult. geschichte.hu-berlin.de/tagungsberichte/id=2723)

Conference Report „History of Robotics“, Annual Meeting of the German Society for the History of Technology (GTG) May 2009, in: Newsletter of the International Committee for the History of Technology ICOHTEC, No. 53, August 2009 (http://www.icohtec.org/_dokumenty/2009-08-icohtecnewsletter-no53.pdf)

Anschriften der Verfasserinnen: Sonja Petersen, Graduiertenkolleg „Topologie der Technik", Technische Universität Darmstadt, Karolinenplatz 5 (Fach 1404), 64289 Darmstadt, E-Mail: Sonja.Petersen@pg.tu-darmstadt.de; Melike Sahinol, Interfakultäres Zentrum für Ethik in den Wissenschaften, Graduiertenkolleg Bioethik, Universität Tübingen, Wilhelmstr. 19, 72074 Tübingen, E-Mail: melike.sahinol@izew.uni-tuebingen.de 
